\title{
Odontologia e saúde mental: experiência do PET Saúde no Centro de Atenção Psicossocial Álcool e Drogas
}

\author{
Franciely Araújo Moura*; Dimitra Castelo Branco**; Thaís de Oliveira Carvalho Granado Santos***; \\ Ana Cláudia Braga Amoras Alves****; Maria Sueli da Silva Kataoka****; Flávia Sirotheau Corrêa \\ Pontes****; Liliane Silva do Nascimento****
}

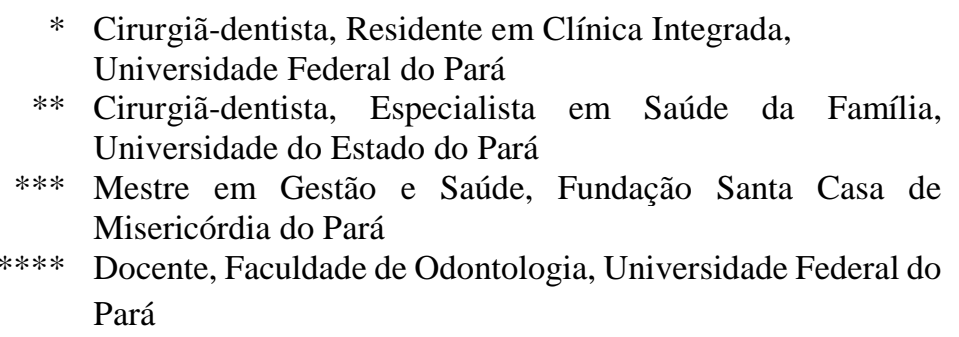

Recebido em 09/08/2018. Aprovado em 07/07/2019.

\begin{abstract}
RESUMO
A fim de discutir a importância de experiências multiprofissionais e o papel de inovação do PET Saúde na formação acadêmica, este artigo relata a experiência do PET - Odontologia da UFPA junto à equipe multiprofissional de um CAPS AD localizado no município de Belém/PA, além de avaliar as condições de saúde bucal dos dependentes químicos. A experiência mostrou a integração entre os diferentes profissionais e motivou o desenvolvimento de habilidades dos discentes para o planejamento de ações integradas e criativas de promoção de saúde, através de metodologias ativas, apontando novos rumos de atenção à saúde, centrado na lógica da realidade da comunidade e não somente no ambiente clínico. As atividades realizadas pela equipe de Odontologia foram integradas às oficinas e grupos desenvolvidos na unidade, como oficinas culinárias e de confecção e o cuidado de hortas, que propiciaram momentos de educação em saúde bucal. Foram realizadas dinâmicas e rodas de conversas, sendo os usuários agentes ativos no processo de construção compartilhada de conhecimento. Ocorreu o "dia D no CAPS AD", no qual os usuários participaram de atividade de escovação supervisionada e foram examinados por equipe médica e odontológica, com coleta de informações clínicas. Considera-se que a interação entre ensino, serviço e comunidade potencializada pelo PET - Saúde conduz a formação em saúde a um novo perfil, em que os profissionais se tornam mais capazes de responder às necessidades dos usuários e atuar de maneira compartilhada nos diferentes cenários.
\end{abstract}

Descritores: Saúde Mental. Integralidade em Saúde. Saúde Bucal. 


\section{INTRODUÇÃO}

O cuidado à saúde mental no Brasil passou por décadas de lutas e modificações até a sua consolidação. Desde os anos 1970 até os dias atuais, várias mudanças ocorreram na forma de acolher e tratar os pacientes com transtornos mentais. Como modelo de substituição aos hospitais psiquiátricos surgiram os Centros de Atenção Psicossocial (CAPS) $)^{1,2}$. O Ministério da Saúde instituiu, pela Portaria $\mathrm{n}^{\circ} 3.088$ de 2011, a Rede de Atenção Psicossocial (RAPS), que propõe um modelo de atenção a pessoas em sofrimento ou transtorno mental e com necessidades decorrentes do uso de crack, álcool e outras drogas no âmbito do Sistema Único de Saúde ${ }^{3}$.

A RAPS articula os vários pontos de atenção à saúde mental, ações e serviços, em diferentes níveis de complexidade ${ }^{3}$. Na Atenção Primária à Saúde (APS), por meio de ações e serviços desenvolvidos por equipes multiprofissionais, principalmente das Unidades Básicas de Saúde (UBS) realiza-se a promoção e prevenção, a redução de danos e o cuidado para pessoas com transtornos mentais e necessidades decorrentes do álcool, crack e outras drogas de modo compartilhado com os demais pontos de atenção ${ }^{1,3}$.

Na RAPS, o ponto de atenção psicossocial especializado é o CAPS que realiza a atenção e cuidado de pessoas com transtornos mentais graves e de casos de necessidades relacionadas ao crack, álcool e outras drogas. Situações não resolvidas somente no âmbito da APS e que demandem maior complexidade tecnológica devem ser referenciadas aos CAPS, compostos por equipe multiprofissional, em regime de tratamento intensivo, semi-intensivo e não-intensivo ${ }^{1,3}$.

A atenção em saúde mental na APS ainda é um desafio que traz impactos para o fluxo de funcionamento da RAPS e para a melhoria da qualidade de vida desses usuários. Importante destacar o papel imprescindível do trabalho nos territórios, a ser desenvolvido fortemente pelas
UBS, na busca ativa, identificação, acolhimento, cuidado e acompanhamento desses usuários, e a adequada referência, quando houver necessidade, aos serviços de maior complexidade, como o CAPS. É, nesse sentido, fundamental que se trabalhe, de fato, a saúde mental ainda na APS e que ocorra o diálogo entre ela e os outros diferentes pontos de atenção da Rede.

Os CAPS Álcool e Drogas (CAPS AD) foram criados em 2002, destinados ao tratamento de pessoas com transtornos decorrentes do uso abusivo de álcool e outras drogas ${ }^{2}$. O cuidado consiste no acolhimento e planejamento terapêutico individualizado, de evolução contínua, com perspectiva integrada, envolvendo a terapia medicamentosa, orientações, realização de grupos e oficinas terapêuticas sob o olhar da política de redução de danos ${ }^{1}$. Essa política envolve práticas de redução dos efeitos globais do uso abusivo de álcool e outras drogas, de modo que o cuidado esteja voltado ao que o usuário deseja e consegue fazer frente ao seu problema ${ }^{4}$.

O uso abusivo de álcool e outras drogas é considerado grave problema de saúde pública. ${ }^{5}$. Estudos mostram a relação e os efeitos do álcool e outras drogas na cavidade oral, os sinais clínicos e alterações encontradas e que têm sido apontadas nos estudos são: lesões por queimaduras, lesões por mordiscamentos, atrição, sinais de bruxismo, descamações, manchamento da língua, doença cárie e doença periodontal generalizada, além do índice de dentes, cariados, perdidos e obturados (CPO-D) geralmente apresentar-se elevado nos estudos com esse público ${ }^{6-8}$.

Considerando-se a relevância do reconhecimento desses agravos em saúde bucal, a integração do cirurgião-dentista à equipe multidisciplinar para a atenção aos dependentes de álcool e drogas torna-se fundamental, porém que ainda deve caminhar bastante para sua efetivação. Deve-se, então, reforçar a atuação da equipe de saúde bucal desde a APS na promoção, prevenção 
e recuperação da saúde mental de forma integrada com os demais profissionais que compõem as equipes das UBS. Dessa maneira, torna-se mais viável a extensão dessa integração multiprofissional, com a inclusão efetiva do cirurgião-dentista, nos demais pontos de atenção da RAPS, aqui destacando-se o CAPS AD.

A integração do cirurgião-dentista no cuidado à saúde mental ainda é um desafio e apresenta muitas lacunas no seu desenvolvimento nos pontos de atenção. Para a transformação dessa realidade, a busca por mudanças no modelo de atenção em saúde bucal é uma importante ferramenta de maneira a atender às reais necessidades de saúde da população. Essa reorientação do modelo de atenção depende e está intimamente relacionada à formação do profissional em Odontologia, que por muito tempo esteve focada primordialmente nos aspectos curativos e restauradores e que não está sendo suficiente para a resolução dos problemas de saúde.

Frente a essas demandas, ressalta-se neste trabalho o Programa de Educação pelo Trabalho para a Saúde (PET - Saúde), que aliado a essas necessidades, aponta para a integração entre ensino, serviço e comunidade como ferramenta capaz de gerar respostas às mudanças no cuidado à saúde e reorientar a formação profissional ${ }^{9}$.

Dessa forma, este estudo objetiva relatar a vivência do PET - Odontologia da Universidade Federal do Pará (UFPA) junto à equipe multiprofissional de um CAPS AD localizado no município de Belém/PA, no período de dezembro de 2016 a agosto de 2017. Além disso, buscou-se avaliar as condições de saúde bucal dos dependentes químicos que participaram da experiência vivenciada.

\section{RELATO DE EXPERIÊNCIA}

Este estudo seguiu os preceitos éticos de pesquisa envolvendo seres humanos, segundo a Resolução 466/2012, sendo submetido e aprovado pelo Comitê de Ética em Pesquisa com Seres Humanos do Instituto de Ciências da Saúde da Universidade Federal do Pará (CEP/ICS), sob CAAE $n^{0}$ 82117416.0.0000.0018 e parecer $n^{0}$ 2.481.506.

\section{A aproximação}

A inserção da Odontologia no CAPS AD se deu em dezembro de 2016, como atividade integrante do grupo PET - Saúde da UFPA. Neste trabalho, acadêmicas de Odontologia foram integradas à equipe de Nutrição já existente na unidade. O primeiro momento da experiência foi de acolhimento das discentes no ambiente do CAPS $\mathrm{AD}$, visando à compreensão do fluxo de assistência, rotina de atendimentos e interação com os demais profissionais presentes na unidade e espaço físico. As ações desenvolvidas foram planejadas de maneira integrada com as outras áreas da saúde atuantes no CAPS AD, em especial com a equipe de Nutrição.

As primeiras atividades realizadas pela equipe de Odontologia foram integradas às oficinas e grupos já desenvolvidos na unidade, como as oficinas culinárias e de confecção e o cuidado de hortas. Dentro dessas oficinas foram propiciados os momentos para a educação em saúde bucal e atividades de promoção de saúde. As oficinas culinárias foram os espaços primordiais para a introdução de questões como a relação da dieta com a saúde bucal. Foram realizadas dinâmicas e rodas de conversas, sendo os usuários agentes ativos no processo de construção compartilhada de conhecimento.

Nas rodas de conversa foram abordados os seguintes temas: dieta cariogênica, dieta não cariogênica e alimentos detergentes. As atividades foram desenvolvidas por meio de metodologias ativas, o que favoreceu a participação do grupo. Além disso, discorreu-se sobre doenças que envolvem a cavidade oral, enfatizando-se a prevenção das doenças cárie e periodontal, com 
enfoque nos meios preventivos de higiene oral.

Além das atividades realizadas durante as oficinas de culinárias, roda de conversa sobre sustentabilidade também foi planejada com a equipe multiprofissional. Por meio de relatos e trocas de experiências, os usuários e profissionais debateram sobre as maneiras de fazer a diminuição da produção de lixo e o reaproveitamento de materiais recicláveis. Nesse espaço realizou-se oficina de criação de porta-escovas confeccionados com garrafas plásticas.

\section{O diagnóstico}

Planejado de maneira integrada, aconteceu o "dia D no CAPS AD", no qual os usuários participaram de atividade de escovação supervisionada e foram examinados por equipe médica e odontológica. Nas consultas odontológicas foram coletadas informações clínicas referentes ao índice CPO-D (somatória de dentes cariados, perdidos e obturados) e sinais clínicos de demais doenças bucais - pigmentação dentária, trauma, sinais de bruxismo e cálculo dentário - foram observados e registrados em fichas individuais. Esses dados foram tabulados e analisados juntamente com variáveis presentes nos prontuários dos usuários.

No total, 22 usuários participaram da atividade. A maioria era do sexo masculino (81,8\%), com idade entre 23 e 72 anos (média=44; desvio padrão=13). Quanto ao tipo de droga utilizada, verificou-se que todos os participantes faziam uso de álcool, 40,9\% utilizavam tabaco, $27,2 \%$ utilizavam maconha, $22,7 \%$ usavam cocaína, $18,1 \%$ faziam uso de crack e $9,09 \%$ usavam oxi. Em relação à associação de drogas, $32 \%$ dos usuários faziam uso de apenas um tipo, $32 \%$ faziam associação de duas drogas e $36 \%$ faziam associação de três ou mais drogas. Quanto ao tempo, a maioria dos usuários $(95,4 \%)$ fazia uso de drogas há mais de 10 anos (tabela 1).

$\mathrm{Na}$ análise dos dados coletados verificou-se que a média do índice CPOD geral da amostra foi de 0,86. Avaliando-se os componentes do índice, separadamente, tem-se que a média de dentes obturados foi de $0,28 \%$, enquanto perdidos e cariados foi de 8,09 e 2,9, respectivamente. As alterações clínicas ainda observadas foram pigmentação dentária, trauma, bruxismo e presença de cálculo dentário; os sinais de bruxismo apareceram em $20 \%$ dos indivíduos, o cálculo dentário estava presente em $50 \%$ da amostra.

\section{A intervenção}

Após a identificação das necessidades de tratamento registradas em fichas de coleta de dados, os pacientes foram encaminhados para atendimento na rede odontológica do município.

A vivência experienciada culminou com a análise dos dados obtidos, a partir dos exames clínicos e dos prontuários dos usuários com vistas a fornecer informações, ainda pouco disponíveis, sobre o perfil da doença cárie em dependentes de álcool e drogas usuários do CAPS AD.

\section{DISCUSSÃO}

O meio político e social nunca foi uma constância, sua modificação se dá com o tempo, momentos históricos e sociais. Dessa forma, a atenção e o cuidado à saúde também sofre modificações, passando, por exemplo, da ótica centrada na doença e meramente terapêutica para uma visão ampliada, e que alcança diversos fatores e atores que são capazes de promover saúde e, também, cidadania, pensamento crítico, inserção social e política, proporcionando autonomia aos indivíduos em seu processo de saúde e adoecimento $^{10}$.

Redescobrir e valorizar o papel expressivo de cada um dos atores no cuidado à saúde profissionais, estudantes e usuários - são desafios lançados na integração entre ensino, serviço e comunidade, que tem no Programa de Ensino pelo Trabalho para a Saúde (PET - Saúde) 
uma importante ferramenta ${ }^{11}$. Nesse contexto, Odontologia, buscou criar espaços abertos de verificou-se que na experiência relatada, a integração entre profissionais e usuários da atuação de equipe de Odontologia no CAPS AD, unidade, preceptores, tutores e alunos vinculados por meio das atividades vinculadas ao PET ao programa.

Tabela 1. Distribuição dos participantes quanto ao sexo, idade, CPO-D, drogas utilizadas e tempo de uso das drogas

\begin{tabular}{|c|c|c|c|c|c|c|c|c|}
\hline Participante & Sexo* & $\begin{array}{l}\text { Idade } \\
\text { (anos) } \\
\end{array}$ & $\begin{array}{c}\text { CPO-D } \\
\text { individual }\end{array}$ & $\mathbf{C}$ & $\mathbf{P}$ & $\mathbf{O}$ & Drogas & $\begin{array}{l}\text { Tempo de } \\
\text { uso (anos) }\end{array}$ \\
\hline 1 & M & 72 & 1.8 & 3 & 17 & 0 & Álcool & $>10$ \\
\hline 2 & M & 47 & 0.39 & 3 & 5 & 1 & Álcool e tabaco & $>10$ \\
\hline 2 & M & 43 & 1.8 & 3 & 17 & 0 & Maconha e Crack & $>10$ \\
\hline 4 & $\mathrm{~F}$ & 30 & 1 & 3 & 12 & 0 & Álcool & $>10$ \\
\hline 5 & M & 23 & 0.36 & 5 & 3 & 0 & Álcool, tabaco, maconha e cocaína & $>10$ \\
\hline 6 & M & 48 & 8.33 & 0 & 25 & 0 & Álcool e tabaco & $>10$ \\
\hline 7 & M & 54 & 1 & 0 & 14 & 0 & Álcool e tabaco & $>10$ \\
\hline 8 & M & 58 & 1.30 & 2 & 15 & 0 & Álcool & $>10$ \\
\hline 9 & M & 60 & 0.86 & 13 & 6 & 0 & Álcool e tabaco & 1 \\
\hline 10 & $\mathrm{~F}$ & 53 & 2 & 5 & 17 & 0 & Álcool & $>10$ \\
\hline 11 & M & 39 & 1.05 & 11 & 9 & 0 & Álcool & $>10$ \\
\hline 12 & M & 36 & 0.11 & 0 & 2 & 1 & Álcool e oxi & $>10$ \\
\hline 13 & M & 31 & 0.35 & 10 & 0 & 0 & Álcool, tabaco e crack & $>10$ \\
\hline 14 & M & 40 & 0.11 & 2 & 1 & 0 & Álcool, tabaco, crack e cocaína & $>10$ \\
\hline 15 & $\mathrm{~F}$ & 57 & 2 & 2 & 18 & 0 & Álcool, maconha, crack, cocaína e oxi & $>10$ \\
\hline 16 & M & 31 & 0 & 0 & 0 & 0 & Álcool, tabaco, maconha e cocaína & $>10$ \\
\hline 17 & M & 33 & 0.30 & 7 & 2 & 0 & Álcool, maconha e crack & $>10$ \\
\hline 18 & M & 42 & 0.19 & 2 & 2 & 1 & Álcool & $>10$ \\
\hline 19 & M & 50 & 0.34 & 2 & 5 & 1 & Álcool e cocaína & $>10$ \\
\hline 20 & M & 36 & 0.40 & 1 & 6 & 2 & Álcool, tabaco e oxi & $>10$ \\
\hline 21 & $\mathrm{~F}$ & 61 & 0 & 0 & 0 & 0 & Álcool & $>10$ \\
\hline 22 & $\mathrm{M}$ & 25 & 0.03 & 1 & 0 & 0 & Álcool, tabaco, maconha, crack e cocaína & $>10$ \\
\hline
\end{tabular}

*M: sexo masculino; F: sexo feminino

Melo et al. $(2016)^{10}$, ao relatarem a experiência de integração entre atividades do PET em uma ESF em Natal/RN, avaliaram que o diálogo entre os saberes acadêmicos e populares têm como grande vantagem a oportunidade do desenvolvimento de habilidades aos discentes de saúde. Essa oportunidade foi bem aproveitada na experiência aqui relatada, pois, diante do cenário, a equipe desenvolveu meios e habilidades para criação e planejamento de atividades diferenciadas e criativas que atendessem à demanda do público.

Em revisão de literatura, Mira et al. $(2016)^{11}$ colocaram a viabilização da interdisciplinaridade como importante impacto do PET Saúde, pelo fato de este permitir a interação entre acadêmicos de diferentes cursos e profissionais inseridos no serviço. Esse aspecto foi vivido na experiência aqui discutida pela integração da equipe de Odontologia 
com as equipes de saúde que fazem parte do CAPS $\mathrm{AD}$ e pelo planejamento integrado das oficinas com a equipe de Nutrição, favorecendo a integralidade e interdisciplinaridade nos temas trabalhados.

Souza et al. $(2014)^{12}$ relataram que a experiência da Odontologia no PET Saúde em duas Unidades de Saúde da Família (USF) de Montes Claros/MG propiciou a inserção da Odontologia nos novos rumos do cuidado à saúde, centrado na lógica da comunidade e não somente no ambiente clínico. Isso pôde ser vivenciado no desenvolvimento deste trabalho, que permitiu a introdução de equipe de Odontologia em uma unidade ligada à RAPS, reforçando-se a importância da articulação da saúde bucal com as RAS.

De acordo com as Diretrizes Curriculares Nacionais para os cursos de Odontologia, entre as habilidades e competências inerentes à formação do cirurgião-dentista, destacam-se a atuação em todos os níveis de atenção à saúde; multiprofissionalidade; articulação da sua atuação com o contexto social dos indivíduos; busca de soluções para os problemas de saúde bucal e necessidades globais da população ${ }^{13}$. Além disso, a experiência do grupo PET- Saúde relatada por Morais et al. (2012) ${ }^{9}$ apontou como um dos grandes resultados a contribuição da integração entre ensino e serviço na formação acadêmica, levando ao surgimento de um novo perfil profissional que desenvolve habilidades e potencialidades para enfrentar limitações, criando soluções e melhorias dos serviços de saúde.

Nesse sentido, os resultados da vivência aqui relatada também refletem diretamente no perfil do profissional e da formação acadêmica em Odontologia, que aponta para reorientação da graduação para as realidades do trabalho em saúde no Brasil, mostrando a relevância da integração ensino-serviço-comunidade nesse processo.

As mudanças na sociedade, no indivíduo e na sua forma de adoecer mostram que são necessárias mudanças na forma de fazer o cuidado em saúde. As práticas fragmentadas de cuidar do ser humano precisam dar espaço às formas criativas, religadas e integradas ${ }^{13}$. A experiência vivenciada no CAPS $\mathrm{AD}$ buscou dar respostas à essa necessidade, elaborando meios, cenários e momentos para promoção e cuidado à saúde através de rodas de conversa, discussões em grupos e oficinas terapêuticas.

As rodas de conversa, pelo uso de relação horizontal de saber e de poder, criam espaços de diálogo compartilhado e construção mútua de conhecimento pela troca de experiências entre os participantes ${ }^{14}$. Melo et al. $(2016)^{10}$ relatam que o emprego dessa metodologia em uma USF trouxe contribuições importantes ao processo de trabalho em saúde, já que reforçou a interação dos participantes, o estabelecimento de vínculo entre equipe e população, o fortalecimento da participação comunitária, o autocuidado e a autonomia dos usuários. Neste relato também foram relevantes as contribuições das rodas de conversa, pois, percebeu-se que a cada atividade os usuários estavam mais integrados, dispostos a dialogar sobre suas vivências e "jogavam na roda" seus conhecimentos sobre os assuntos abordados. Dessa forma, o conhecimento pôde ser construído com a participação de todos os envolvidos.

Em seu estudo sobre o instrumento roda de conversa no cuidado à saúde mental, Costa et al. $(2015)^{14}$ observaram que com essa metodologia obteve-se melhor desempenho na comunicação interpessoal dos indivíduos, maior capacidade na resolução de conflitos frente aos desafios da sociedade e consequente redução de agravos relacionados aos sofrimentos psíquicos. Usar o mecanismo da roda de conversa na abordagem de assuntos que promovem saúde, dentro das necessidades encontradas no CAPS $\mathrm{AD}$, atende às perspectivas da Rede de Atenção Psicossocial, que tem como uma de suas diretrizes a diversificação 
das estratégias de cuidado $^{3}$. Nesse espaço mais diversificado no cuidado em saúde, o conhecimento deixa de estar centralizado no profissional, como acontece tradicionalmente, passando a ser construído e compartilhado.

Parte das atividades relatadas neste trabalho ocorreu entre grupos e oficinas terapêuticas, entre elas a oficina de horta, as oficinas culinárias e a oficina de confecção de métodos alternativos de higiene oral e portas escova de garrafas plásticas. As oficinas terapêuticas, assim como as rodas de conversa, destacam-se no novo modelo de cuidado aos transtornos mentais, elas podem ser consideradas instrumentos clínicos e políticos, que favorecem a expressão e a autonomia ${ }^{16}$.

De acordo com Souza e Pinheiro (2012) ${ }^{17}$, as oficinas terapêuticas permitem o trabalho na ótica da redução de danos, favorecem a corresponsabilidade e torna o usuário ativo em seu processo de saúde. Nas oficinas realizadas no CAPS AD, especialmente as oficinas culinárias e a oficina de confecção de porta escova de garrafa plástica, os usuários se encontraram diante da possibilidade de intervir ativamente na construção de algum produto. No contexto terapêutico, esse processo foi de fundamental importância, por possibilitar a coparticipação em saúde.

Além das intervenções de educação e promoção de saúde, como etapa transversal durante toda a experiência da Odontologia na unidade, o momento de avaliação clínica dos usuários foi importante para a coleta de dados, para conhecimento do perfil de saúde bucal dos dependentes químicos em tratamento.

Assim como em estudos anteriores, neste trabalho o sexo masculino foi apontado como o mais frequente entre os usuários de álcool e outras drogas $^{6,7,18}$. A droga predominantemente consumida pelos usuários do CAPS $\mathrm{AD}$ foi $\mathrm{o}$ álcool, os resultados de Costa et al. $(2011)^{18}$ e de Albini et al. $(2015)^{8}$ também revelaram o mesmo resultado. No presente estudo houve grande prevalência de usuários há mais de 10 anos, variável importante a ser analisada, pois alguns estudos revelaram que quanto maior o tempo de dependência, mais afetada era a saúde bucal dos indivíduos e maior era o índice de CPOD encontrado $^{8,18}$.

Romão et al. $(2016)^{19}$ apontam índices CPOD elevados nessa população, sendo os componentes cariado e perdido os mais relevantes. Os resultados do SB Brasil 2010, revelaram índice de CPOD de 16,75 para a população na faixa etária de 35 a $44 \operatorname{anos}^{20}$. A idade média dos indivíduos deste estudo foi 44,2 anos, com índice de CPOD consideravelmente mais baixo $(1,006)$ do que a média nacional. Em estudos que traçaram o perfil epidemiológico de dependentes químicos foram observados valores elevados do índice CPOD, sendo os componentes cariado e perdido os mais altos $^{7,8}$. No relatório do SB Brasil 2010 destacou-se que o componente perdido foi responsável por cerca de $44,7 \%$ do índice no grupo de 35 a 44 $\operatorname{anos}^{20}$. Esses resultados estão em consonância com os achados deste estudo, os componentes cariado e perdido da amostra apresentaram-se consideravelmente altos. Falcão et al. $(2015)^{7}$ observaram que mais da metade dos usuários atendidos em um CAPS AD apresentavam cálculo supra e/ou subgengival e que um número considerável mostrava sinais de bruxismo, agravos que também foram evidenciados entre os usuários participantes desta experiência.

\section{CONSIDERAÇÕES FINAIS}

A experiência do PET- Odontologia em uma unidade especializada da Rede de Atenção Psicossocial mostrou o potencial da integração entre ensino, serviço e comunidade na produção de novos métodos de cuidado à saúde, oportunizando a criação de metodologias que respondam às necessidades de saúde da comunidade. A exploração de novos cenários compartilhados com outros profissionais e áreas foi importante, 
apontando para a necessidade de mudança na formação em Odontologia integrada e com atuação em diferentes ambientes, não se limitando ao isolamento e atuação meramente clínica.

\section{ABSTRACT \\ Odontology and mental health: experience of PET Saúde in the Center for Psychosocial Care Alcohol and Drugs}

In order to discuss the importance of multiprofessional experiences and the innovation role of the PET - Saúde in academic training, this article reports on the experience PET - Odontology of UFPA with the multi-professional team of a CAPS $\mathrm{AD}$ located in the city of Belém / PA, besides evaluating the oral health conditions of chemical dependents. The experience showed the integration among the different professionals and motivated the development of the students' abilities to the planning of integrated and creative actions of health promotion, through active methodologies, pointing out new directions of health care, centered on the logic of the community reality and not only in the clinical environment. The activities carried out by the Dentistry team were integrated with the workshops and groups developed at the unit, such as cooking and cooking workshops and garden care, which provided moments of education in oral health. Was made dynamics and wheels of conversations, and the users are active agents in the process of shared knowledge construction. "D-day at CAPS AD" occurred, in which users participated in supervised brushing activity and were examined by medical and dental staff, collecting clinical information. It is considered that the interaction between teaching, service and community enhanced by PET - Saúde leads health education to a new profile in which professionals become better able to respond to users' needs and act in a shared way in different scenarios.

Descriptors: Mental Health. Integrality in Health. Oral Health.

\section{REFERÊNCIAS}

1. Brasil. Ministério da Saúde. Secretaria de Atenção à Saúde. Departamento de Ações
Programáticas Estratégicas. Saúde Mental no SUS: Os Centros de Atenção Psicossocial. Departamento de Ações Programáticas Estratégicas. Brasília: Ministério da Saúde, 2004. $86 \mathrm{p}$.

2. Larentis CP, Maggi A. Centros de Atenção Psicossocial Álcool e Drogas e a Psicologia. Aletheia. 2012; 37: 121-32.

3. Brasil. Ministério da saúde. Portaria $n^{\circ} 3088$, de 23 de dezembro de 2011. Institui a Rede de Atenção psicossocial para pessoas com sofrimento ou transtorno mental e com necessidades decorrentes do uso de crack, álcool e outras drogas, no âmbito do Sistema Único de Saúde. Diário Oficial da União 2011; dez 26.

4. Brasil. Ministério da Saúde. Secretaria de Atenção à Saúde. Atenção Básica. Saúde mental. Cadernos de Atenção Básica. Brasília: Ministério da Saúde, 2013. 176 p.

5. United Nations Office on Drugs and Crime. Relatório Mundial sobre Drogas 2017 [Acesso em 05 mar 2018]. Disponível em: http://www.unodc.org/.

6. Cima T, Corrêa K. Alterações bucais em dependentes químicos. J Oral Invest. 2014; 3(2): 8-12.

7. Falcão CAM, Santos RO, Pereira RMS, Silva TSO, Ferreira RS, Silva FWC, et al. Saúde bucal em dependentes químicos. Rev Interd Ciên Saúde. 2015:112-19.

8. Albini MB, Couto ACF, Invernici MM, Martins MC, de Lima AAS, Gabardo MCL, et al. Perfil epidemiológico e condição bucal de usuários de drogas em dois municípios do Estado do Paraná, Brasil. Rev Odontol UNESP. 2015; 44(4): 244-9.

9. Morais FRR, Jales GML, da Silva MJC, Fernandes SF. A importância do PET Saúde para a formação acadêmica do enfermeiro. Trab Educ Saúde. 2012;10 (3): 541-51.

10. de Melo RHV, Felipe MCP, da Cunha ATR, 
de Vilar RLA, Pereira EJS, Carneiro NEA, et al. Roda de conversa: uma articulação solidária entre ensino, serviço e comunidade. Rev Bras Educ Méd. 2016; 40(2):301-9.

11. Mira QLM, Barreto RMA, Vasconcelos MIO. Impacto do PET - Saúde na formação profissional: uma revisão integrativa. Rev Baiana Saúde Pública. 2016; 40(2):514-31.

12. de Souza GCLL, Lopes MRL, Rodrigues CAQ, Moreira KS, Oliveira MP, Morais AJD, et al. O PET - Saúde na formação acadêmica em odontologia: contribuições e perspectivas. Rev ABENO. 2014; 14(1):73-80.

13. Brasil. Conselho Nacional de Educação. Câmara de Educação Superior. Resolução CNE/CES 3, de 19 de fevereiro de 2002. Institui Diretrizes Curriculares Nacionais do Curso de Graduação em Odontologia. 2002.

14. Costa RRO, Filho JB, de Medeiros SM, da Silva MBM. As rodas de conversa como espaço de cuidado e promoção de saúde mental. Rev de Atenção à Saúde. 2015 janmar; 13 (43): 30-36.

15. Sampaio J, Santos GC, Agostini M, salvador As. Limites e potencialidades das rodas de conversa no cuidado à saúde: uma experiência com jovens no sertão pernambucano. Interface Comun Educ Saúde. 2014; 18(1):1299-312.
16. Ibiapina ARS, Monteiro CFS, Alencar DC, Fernandes MA, Filho AAIC. Oficinas terapêuticas e as mudanças sociais em pacientes com transtorno mental. Esc Anna Nery Rev Enferm. 2017; 21 (3): e20160375.

17. Souza LGS, Pinheiro LB. Oficinas terapêuticas em um Centro de Atenção Psicossocial - álcool e drogas. Aletheia. 2012; 38(39):2018-227.

18. da Costa SKP, Godoy GP, de Gomes DQ, Pereira JV, Lins RDAC. Fatores sociodemográficos e condições de saúde bucal em drogaditos. Pesq Bras Odontoped Clin Integr. 2011;11(1):99-104.

19. Romão DA, Falcão A, Pereira AC, Brizon VSC. Experiência de cárie dentária em usuários de drogas psicoativas. Rev Bras Pesq Saúde. 2016; 18(3):137-143.

20. Brasil. Ministério da Saúde. SB Brasil 2010: Pesquisa Nacional de Saúde Bucal: resultados principais. Brasília: Ministério da Saúde, 2012.

\section{Correspondência para:}

Liliane Silva do Nascimento

e-mail: 1ilianenascimento2001@ gmail.com

Praça Camilo Salgado, 1, Umarizal

66.050-160 Belém/PA 